\title{
SIMPLIFIED DIGITAL HOLOGRAPHIC RECONSTRUCTION USING STATISTICAL METHODS
}

\section{Jeffrey A. Fessler, Senior Member, IEEE}

University of Michigan

Dept. of Electrical Engineering and Computer Science

Ann Arbor, MI 48109, USA
Saowapak Sotthivirat

\author{
National Electronics and Computer Technology Center \\ Ministry of Science and Technology \\ Klong Luang, Pathumthani 12120, THAILAND
}

\begin{abstract}
For reconstructing a complex object wavefront from digital holograms, we propose a new penalized-likelihood approach based on the measurement statistics and edge-preserving regularization. The log-likelihood is complicated since the measurements are related to the magnitude of the complex beam. We use optimization transfer to derive a new simplified iterative algorithm that monotonically decreases the cost function. Unlike the conventional FFT-based holographic reconstruction method, the new approach uses all of the measured data, and can be applied to holograms with any (known) reference beam pattern. Simulation results demonstrate the potential for improved image quality.
\end{abstract}

\section{INTRODUCTION}

In digital holography, the interference between a reference beam and a wavefront from an object of interest is recorded by an electronic sensor such as a CCD array. Under appropriate sampling conditions, one can reconstruct the complex object wave from the digital hologram. The classical computer reconstruction method for off-axis holograms is to compute the 2D FFT of the measured hologram, use a window to select the small portion of the spectrum corresponding to the appropriate interference term, shift that portion to DC, zero pad, and then take the inverse 2D FFT. (The method is a 2D analog of demodulation of AM audio signals.) This simple approach is applicable only to plane wave reference beams, uses only a small fraction of the measured data, yet can still suffer from interference from the other terms, and accounts for noise only implicitly by spectral apodization. The potential use of digital holography for biological microscopy has renewed interest in finding improved reconstruction methods for digital holograms,e.g., [1].

We recently proposed a new numerical reconstruction approach [2] formulated from first principles including the 60711 .

Supported in part by NSF grant BES-9982349 and NIH grant CA- physical optical model and a statistical model for measurement noise. The problem is ill-posed, so we perform penalizedlikelihood estimation using edge-preserving regularization. The log-likelihood is quite complicated since the object wave is complex whereas the measurements are related to the magnitude of the sum of the object beam and the reference beam. Using optimization transfer techniques, we derived in [2] an iterative algorithm that monotonically decreases the cost function each iteration and thus typically converges to a local minimizer. The computation per iteration is comparable to the EM algorithm for image restoration. The approach can be applied to holograms with any (known) reference beam pattern, including the types of spherical patterns seen in practice. Simulation results show that this statistical approach has the potential to improve image quality in digital holography relative to conventional reconstruction methods. In this paper, we derive a simpler iterative algorithm for this problem.

\section{THEORY}

As described in detail in [2], the problem of image reconstruction in digital holography can be posed as the following penalized-likelihood estimation problem:

$$
\begin{gathered}
\hat{x}=\underset{\boldsymbol{x} \in \mathbb{C}^{M}}{\arg \min } \Psi(\boldsymbol{x}) \\
\Psi(\boldsymbol{x})=\ell(\boldsymbol{x})+R(\boldsymbol{x}),
\end{gathered}
$$

where $\boldsymbol{x}=\left(x_{1}, \ldots, x_{M}\right)$ denotes the vector of unknown pixel values in the object to be recovered, $\ell(x)$ denotes the negative log-likelihood, and $R(x)$ denotes a roughness penalty function. We assume a Poisson statistical model for the measurements:

$$
\begin{aligned}
y_{i} & \sim \text { Poisson }\left\{y_{i}(\boldsymbol{x})\right\}, \quad i=1, \ldots, N \\
y_{i}(\boldsymbol{x}) & =\left|[\boldsymbol{A} \boldsymbol{x}]_{i}+u_{i}\right|^{2}+r_{i},
\end{aligned}
$$

where $\boldsymbol{A} \in \mathbb{C}^{N}{ }^{M}$ denotes the system matrix that models the imaging system, $u_{i} \in \mathbb{C}$ denotes the $i$ th sample of the 
reference beam, $r_{i} \in[0, \infty)$ denotes the additive effect of detector dark current and possible gaussian readout noise [3], $y_{i} \in \mathbb{R}$ denotes the $i$ th element of the hologram measurement (e.g., recorded by a CCD detector) and

$$
[\boldsymbol{A} \boldsymbol{x}]_{i}=\sum_{j=\mathbf{1}}^{M} a_{i j} x_{j}
$$

We assume that $\boldsymbol{A},\left\{u_{i}\right\},\left\{r_{i}\right\},\left\{y_{i}\right\}$, are all known, and the goal is to determine the image pixel values $\boldsymbol{x}$. For independent measurements, the corresponding negative loglikelihood is

$$
\begin{gathered}
\qquad(\boldsymbol{x})=\sum_{i=1}^{N} h_{i}\left([\boldsymbol{A} \boldsymbol{x}]_{i}+u_{i}\right) \\
h_{i}(z)=|z|^{2}+r_{i} \quad y_{i} \log |z|^{2}+r_{i} .
\end{gathered}
$$

The minimization problem (1) is challenging because $\boldsymbol{x}$ is complex and $h_{i}()$ is non-quadratic. One can show that the (column) gradient of $t(x)$ is

$$
\nabla \mathfrak{E}(\boldsymbol{x})=\boldsymbol{A}^{\prime} \operatorname{diag}\left\{2\left(1 \frac{y_{i}}{y_{i}(\boldsymbol{x})}\right)\right\}(\boldsymbol{A x}+\boldsymbol{u}),
$$

where " " " denotes Hermitian transpose. One could attempt to find the minimizer $\hat{\boldsymbol{x}}$ by applying a gradient descent algorithm of the form

$$
\boldsymbol{x}^{(n+1)}=\boldsymbol{x}^{(n)} \quad \alpha \nabla \Psi\left(\boldsymbol{x}^{(n)}\right) .
$$

However, the conventional "trial and error" approach to choosing the step size $\alpha$ is inconvenient; in general this approach is not guaranteed to monotonically decrease the cost function so divergence is possible. The dominant computation for each iteration of any such algorithm is the gradient (6); this requires one multiplication by $\boldsymbol{A}$ and by $\boldsymbol{A}^{\prime}$. This is comparable to the EM algorithm for image restoration.

In [2], we proposed to apply the optimization transfer principle to solve this minimization problem [4]. For each iteration $n$, we find a surrogate function $\phi^{(n)}$ that satisfies the two conditions:

$$
\begin{aligned}
\phi^{(n)}\left(x^{(n)}\right) & =\Psi\left(x^{(n)}\right) \\
\phi^{(n)}(x) & \geq \Psi(x) .
\end{aligned}
$$

These majorization conditions ensure that the optimization transfer algorithm

$$
\boldsymbol{x}^{(n+1)}=\underset{\boldsymbol{x} \in \mathbb{C}}{\arg \min } \phi^{(n)}(\boldsymbol{x})
$$

will monotonically decrease the cost function, i.e.,

$$
\Psi x^{(n+1)} \leq \Psi\left(x^{(n)}\right) .
$$

Since the cost function is nonconvex, such monotonic methods typically will converge to a local minimizer near the initial guess $x^{(0)}$.

In this paper, we describe an alternative approach that may be somewhat simpler. Given a current guess $x$, let the (negative) gradient define a search direction

$$
d=\nabla \Psi(x)
$$

and consider the ID "line search" minimization problem

$$
\begin{gathered}
\alpha^{(n)}=\underset{\alpha}{\arg \min } f(\alpha) \\
f(\alpha)=\Psi(\boldsymbol{x}+\alpha \boldsymbol{d}) .
\end{gathered}
$$

We propose to apply the principles of optimization transfer to this ID minimization problem. (An algorithm for a simpler problem that employed a similar ID search strategy was described in [5].) Note that

$$
f(\alpha)=\sum_{i=1}^{N} g_{i}(\alpha)+R(\boldsymbol{x}+\alpha \boldsymbol{d})
$$

where

$$
g_{i}(\alpha) \triangleq h_{i}\left([\boldsymbol{A}(\boldsymbol{x}+\alpha \boldsymbol{d})]_{i}+u_{i}\right)
$$

and

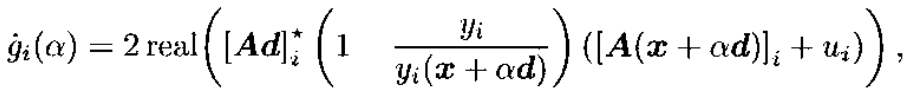

where "^" denotes conjugate. The key idea here is that the functions $g_{i}()$ have bounded curvature, so it is possible to find quadratic surrogate functions of the form

$q_{i}\left(\alpha ; \alpha^{\prime}\right)=g_{i}\left(\alpha^{\prime}\right)+\dot{g}_{i}\left(\alpha^{\prime}\right)\left(\alpha \quad \alpha^{\prime}\right)+\frac{1}{2} \breve{c}_{i}\left(\alpha^{\prime}\right)\left(\alpha \quad \alpha^{\prime}\right)^{2}$

where the curvatures $\breve{c}_{i}$ are chosen such that

$$
q_{i}\left(\alpha ; \alpha^{\prime}\right) \geq g_{i}(\alpha) \text {. }
$$

The condition $q_{i}\left(\alpha^{\prime} ; \alpha^{\prime}\right)=g_{i}\left(\alpha^{\prime}\right)$ is satisfied by construction. Similarly one can find a quadratic surrogate function for $R(\boldsymbol{x}+\alpha \boldsymbol{d})$, call it $q_{0}\left(\alpha ; \alpha^{\prime}\right)$, with curvature $\breve{c}_{0}\left(\alpha^{\prime}\right)$ $[6,7]$. Then we have the overall quadratic surrogate for $f(\alpha)$ constructed as follows:

$$
q\left(\alpha ; \alpha^{\prime}\right)=\sum_{i=0}^{N} q_{i}\left(\alpha ; \alpha^{\prime}\right) .
$$

We can minimize this surrogate analytically, leading the following "sub-iteration" for finding the minimizing value of $\alpha$ :

$$
\begin{aligned}
& \alpha^{\text {new }}=\underset{\alpha}{\arg \min } q\left(\alpha ; \alpha^{\text {old }}\right)=\alpha^{\text {old }} \frac{\dot{q}\left(\alpha^{\text {old }} ; \alpha^{\text {old }}\right)}{\ddot{q}\left(\alpha^{\text {old }} ; \alpha^{\text {old }}\right)} \\
& =\alpha^{\text {old }} \frac{\dot{f}\left(\alpha^{\text {old }}\right)}{\sum_{i=0}^{N} \breve{c}_{i}\left(\alpha^{\text {old }}\right)} .
\end{aligned}
$$


Being based on the optimization transfer principle, this subiteration is guaranted to decrease $f()$ monotonically. We initialize it with $\alpha^{\text {old }}=0$, which then ensures that when the resulting $\alpha^{\text {new }}$ (after one or more sub-iterations) is used within the overall iteration (7), the overall algorithm is monotonic.

\section{CURVATURES}

It remains to find curvatures for (8) that ensure the majorization condition (9). Using (5) and (4), we have that

$$
g_{i}(t)=m_{i}(t) \quad y_{i} \log m_{i}(t)
$$

where

$$
\begin{aligned}
m_{i}(t) & \triangleq\left|[\boldsymbol{A}(\boldsymbol{x}+t \boldsymbol{d})]_{i}+u_{i}\right|^{2}+r_{i}=\left|l_{i}+t p_{i}\right|^{2}+r_{i} \\
& =t^{2}\left|p_{i}\right|^{2}+2 t \operatorname{real}\left(l_{i}^{\star} p_{i}\right)+\left|l_{i}\right|^{2}+r_{i} \\
l_{i} & \triangleq[\boldsymbol{A} \boldsymbol{x}]_{i}+u_{i}, p_{i} \triangleq[\boldsymbol{A} \boldsymbol{d}]_{i} .
\end{aligned}
$$

For brevity we also write

$$
\begin{aligned}
& m_{i}(t)=w_{i} t^{2}+2 b_{i} t+v_{i} \\
& \dot{m}_{i}(t)=2\left(w_{i} t+b_{i}\right),
\end{aligned}
$$

where $w_{i} \triangleq\left|p_{i}\right|^{2}, b_{i} \triangleq \operatorname{real}\left(l_{i}^{\star} p_{i}\right)$, and $v_{i} \triangleq r_{i}+\left|l_{i}\right|^{2}$. The derivatives of $g_{i}$ are:

$$
\begin{aligned}
& \dot{g}_{i}(t)=1 \frac{y_{i}}{m_{i}(t)} \dot{m}_{i}(t) \\
& \ddot{g}_{i}(t)=1 \frac{y_{i}}{m_{i}(t)} \ddot{m}_{i}(t)+\frac{y_{i}}{m_{i}^{2}(t)} \dot{m}_{i}^{2}(t) .
\end{aligned}
$$

It is shown in [2] that the following expression is a suitable choice for the curvature that will ensure majorization:

$$
\breve{c}_{i}(s)=\underset{t \neq s}{\arg \max } \delta_{i}(t ; s), \quad \delta_{i}(t ; s) \triangleq \frac{\dot{g}_{i}(t) \dot{g}_{i}(s)}{t s},
$$

provided the maximum is positive and finite. Considering the particular form of $m_{i}(t)$ in this problem, we can solve for $\breve{c}_{i}(s)$ analytically.

Hereafter we drop the subscript "i" for simplicity. Because $\dot{m}(t)$ is linear in $t$, one can show that

$$
\delta(t ; s)=2 w+\frac{y}{m(s)} \frac{m(t) \dot{m}(s) \quad m(s) \dot{m}(t)}{(t \quad s) m(t)} .
$$

Elementary simplifications lead to

$\frac{m(t) \dot{m}(s) \quad m(s) \dot{m}(t)}{t \quad s}=2\left[w b(t+s)+w^{2} s t+2 b^{2} \quad w v\right]$.

Extrema of $\delta(; s)$ occur at the zeros of its derivative with respect to $t, i . e .$, where

$$
0=\frac{\partial}{\partial t} \frac{m(t) \dot{m}(s) m(s) \dot{m}(t)}{(t \quad s) m(t)}
$$

or equivalently

$$
\begin{aligned}
& m(t)\left[w b+w^{2} s\right]=\left[w b(t+s)+w^{2} s t+2 b^{2} \quad w v\right] \dot{m}(t) \\
& 0=w\left(w b+w^{2} s\right) t^{2}+2 w\left(w b s+2 b^{2} \quad w v\right) t \\
& +2 b^{2}(w s+2 b) \quad v w(w s+3 b) \text {. }
\end{aligned}
$$

This is a quadratic formula in $t$, so one can easily find its roots. One can then check the values of $\delta$ at each root. Since $m_{i}$ is a quadratic function of $t$, the curvature $\ddot{g}_{i}(t)$ is a rational function of $t$, and one can show that it is bounded. So the root corresponding to the larger (necessarily finite) value of $\delta$ will be an appropriate choice for $\breve{c}_{i}$. Typically, these polynomial manipulations require much less computation than computing the cost function gradient (6).

\section{SIMULATION RESULTS}

Fig. 1a shows a simulated 1D hologram generated according to the model (2) for the case where $\boldsymbol{A}$ corresponds to convolution with a 3-point moving average filter, for the complex signal shown in Fig. 1 b.

Fig. 2a shows the conventional reconstruction obtained by windowing one of the sidelobes of the spectrum of the recorded hologram. This estimate is noisy and has ringing.

Fig. $2 b$ shows the proposed penalized-likelihood estimate $\hat{x}$ using edge-preserving regularization. The NRMS error of this approach is about $7 \%$, compared to $14 \%$ for the conventional approach.

Fig. 3 shows the conventional and proposed penalizedlikelihood reconstruction for a $2 \mathrm{D}$ complex object using the same setup as in [2]. The NRMS error is about $14 \%$ for the proposed approach and $40 \%$ for the conventional approach.
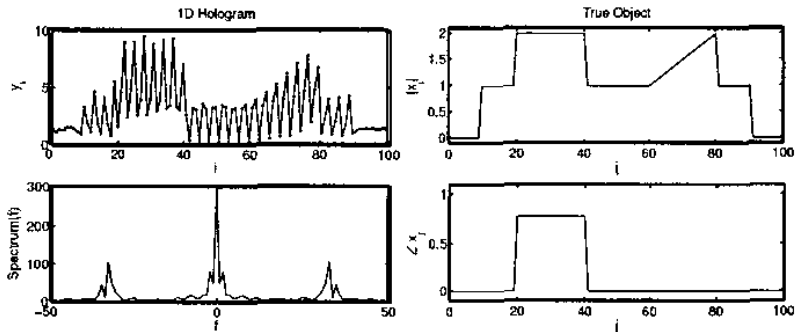

a) 10 hologram and its spectrum

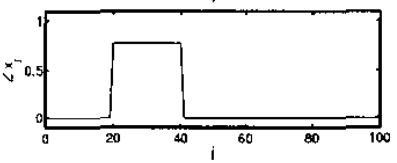

b) Magnitude and phase of object

Fig. 1. Simulated ID hologram data and true object.

\section{DISCUSSION}

We have described an algorithm for penalized-likelihood reconstruction in for digital holography that is an alternative to the method described in [2]. As in [2], this approach 

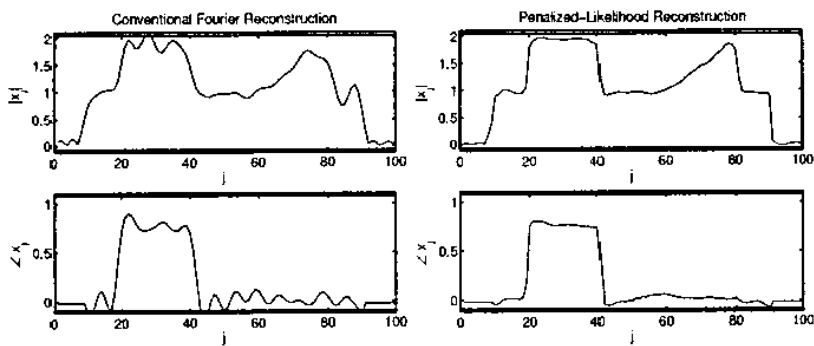

a) Conventional reconstruction

b) Proposed reconstruction

Fig. 2. Magnitude and phase of estimate $\hat{\boldsymbol{x}}$.
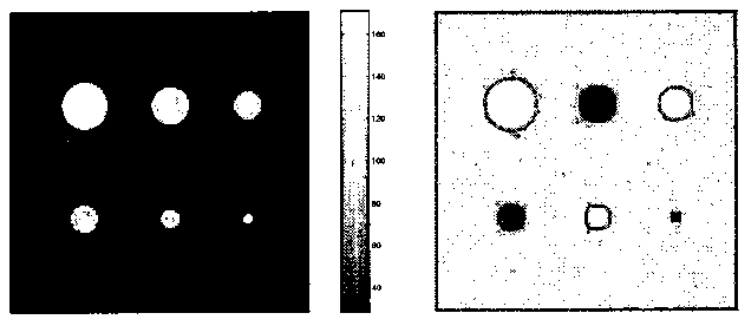

a) Magnitude and phase of true object
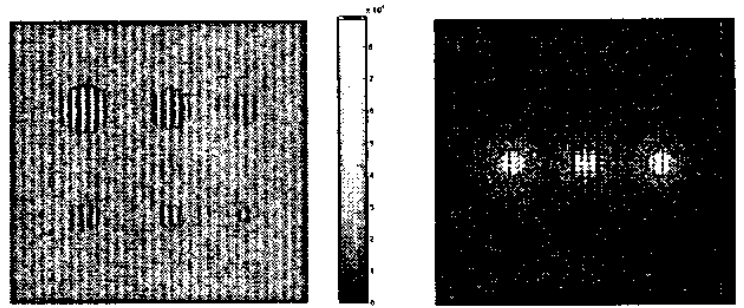

b) Hologram and its spectrum
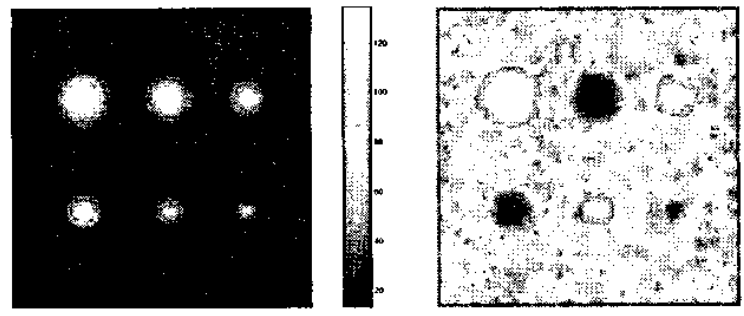

c) Magnitude and phase of FFT-based reconstruction
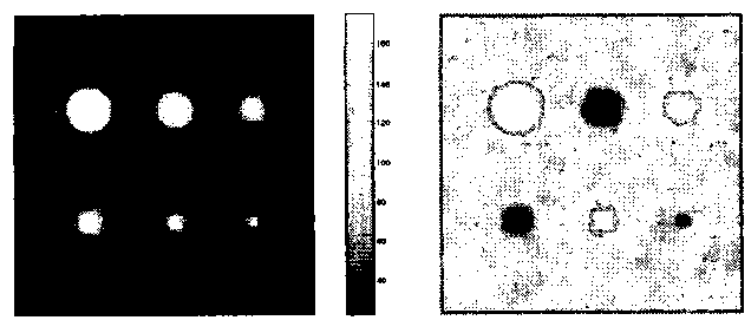

d) Magnitude and phase of proposed reconstruction

Fig. 3. Simulation and reconstruction of $2 \mathrm{D}$ complex object. can improve the quality of the image relative to the conventional FFT-based holographic reconstruction technique. Moreover, unlike the conventional approach, our statistical reconstruction is not limited by the assumption of a planar reference beam.

\section{Acknowledgment}

The authors acknowledge the contributions of Brian Athey, Emmett Leith, and Kurt Mills.

\section{REFERENCES}

[1] M. Liebling, T. Blu, E. Cuche, P. Marquet, C. Depeursinge, and $M$. Unser, "A novel non-diffractive reconstruction method for digital holographic microscopy," in Proc. IEEE Int'l Symp. on Biomedical Imaging, 2002, pp. 625-628.

[2] S. Sotthivirat and J. A. Fessler, "Penalized-likelihood image reconstruction for digital holography," J. Opt. Soc. Am. A, vol. 21, no. 5, pp. 737-750, May 2004.

[3] D. L. Snyder, C. W. Helstrom, A. D. Lanterman, M. Faisal, and R. L. White, "Compensation for readout noise in CCD images," J. Opt. Soc. Am. A, vol. 12, no. 2, pp. 272-83, Feb. 1995.

[4] K. Lange, Numerical analysis for statisticians, Springer-Verlag, New York, 1999.

[5] J. A. Fessler and S. D. Booth, "Conjugate-gradient preconditioning methods for shift-variant PET image reconstruction," IEEE Tr. Im. Proc., vol. 8, no. 5, pp. 688-99, May 1999.

[6] J. A. Fessler, "Statistical image reconstruction methods for transmission tomography," in Handbook of Medical Imaging, Volume 2. Medical Image Processing and Analysis, M. Sonka and J. Michael Fitzpatrick, Eds., pp. 1-70. SPIE, Bellingham, 2000.

[7] J. A. Fessler, "Grouped coordinate descent algorithms for robust edge-preserving image restoration," in Proc. SPIE 3I70, Im. Recon. and Restor. II, 1997, pp. 184-94. 